\title{
HERNÁN RUIZ EL JOVEN Y LA TORRE DE SANTA MARÍA DE LA MOTA DE MARCHENA
}

\author{
POR ALFREDO J. MORALES
}

A finales de mayo de 1562 Hemán Ruiz el Joven era designado maestro mayor del arzobispado hispalense ${ }^{\prime}$. Para el arquitecto, tal nombramiento suponía un paso decisivo en su carrera profesional, a la vez que una satisfación a su incontenible ambición. Para las autoridades eclesiásticas, su presencia en las numerosas empresas constructivas que patrocinaban eran, por un lado, señal de reconocimiento a la ingente y fecunda actividad desarrollada, tanto en la propia Sevilla como en las tierras de su archidiócesis, desde su incorporación a las obras de la catedral sevillana, ocurrida cinco años antes ${ }^{2}$. Por otro, era expresión de confianza en su magisterio a la hora de subsanar los fallos y problemas que se habían detectado en numerosas fábricas, debidos tanto a la carencia de maestros en su dirección, como a la incapacidad de quienes las gobermaban.

Las claúsulas del contrato de nombramiento referido hacían al arquitecto responsable de trazar, administrar y dar orden a los edificios enumerados en el mismo o que se pudieran iniciar en el futuro, obligándole a visitarlos al menos una vez al año, si bien debería acudir a sus obras cada vez que se le convocase o cuando el creyera oportuno. También le correspondía al maestro la designación de los aparejadores y asentadores de las distintas fábricas, haciéndosele responsable de los daños que se ocasionaran en ellas por defectos de las trazas o por falta de control de los procesos constructivos. En atención a su ocupación y dependiendo de la importancia de las poblaciones y templos, el arquitecto debía recibir unas cantidades anuales, además de pagársele por cada una de las visitas de inspección que realizase.

A pesar de la amplia relación de iglesias que figura en el documento, el trabajo del arquitecto no se circunscribió a las mismas. De hecho, antes de 1562 ya había

1. El documento correspondiente fue publicado por LOPEZ MARTINEZ, Celestino: Desde Jerónimo Hernández hasta Martínez Montañés. Sevilla, 1929. Págs. 150-151.

2. Véase MORALES, Alfredo J.: La Capilla Real de Sevilla. Sevilla, 1979. Pág. 46. 
intervenido en algunas iglesias del arzobispado hispalense ${ }^{3}$. Por otra parte, despues de la fecha mencionada y con las condiciones estipuladas en el contrato se le encomendaron obras en otros templos de la archidiócesis ${ }^{4}$. Por cierto que entre las localidades e iglesias que se incluyen en el citado documento no se cita ninguna de las de Marchena, lo cual parece indicar que en esa fecha, 1562, no se llevaban a cabo obras en ellas. No obstante, cinco años más tarde ya se menciona al arquitecto en relación con las torres de las iglesias de San Juan y San Miguel de dicha población de la campiña sevillana ${ }^{5}$. Esto parece indicar que entre las dos fechas citadas se encomendó a Hernán Ruiz algún tipo de trabajo relacionado con ellas. La vaguedad y brevedad del apunte que se incluye en el libro de cuentas parroquial impide precisar el alcance de su intervención, aunque por la expresión textual, "porlavenida que vino avisitar la torre de San Juan eSan Miguel", también pudiera pensarse que el objetivo de su viaje fuera inspeccionar el estado de conservación de ambas torres ${ }^{6}$. De hecho, ninguna otra referencia documental posterior alude a obras en los mencionados campanarios.

Por el contrario, las fuentes documentales tanto del mismo año 1567 como posteriores, vinculan a Hernán Ruiz el Joven con otro templo marchenero, el de Santa María de la Mota, situado junto al palacio de los Duques de Arcos y Señores de Marchena, en la colina que domina la población ${ }^{7}$. La primera noticia, sin precisar mes concreto aunque presumiblemente correspondiente a uno del primer trimestre, se refiere a un pago de 36 reales efectuado al arquitecto "dela visita que hizo enla obra dela torre de Nuestra Señora y del parecer que dio para acrecentarla yglesia" 8 . De la frase transcrita se deducen dos cuestiones importantes. Por una lado, que se estaba trabajando en la torre de la iglesia. Por otro, que se proyectaba ampliar el templo. De este último asunto, la falta de posterior información parece indicar que no se pasó del proyecto, tal vez por la opinión contraria del maestro mayor. Sin embargo, respecto a la torre, diversos pagos anotados en el libro de cuentas con

3. Sirvan de ejemplo las correspondientes a la Iglesia de Santo Domingo de Bornos (Cádiz), iniciadas en 1559, o las emprendidas un año antes en la parroquia de la población sevillana de Peñaflor, que no se han conservado. Véase LOPEZ MARTINEZ, Celestino : Op. Cit. Pág. 132 y LOPEZ MARTINEZ, Celestino: El arquitecto Hernan Ruiz en Sevilla. Sevilla, 1949. Págs. 51-52, respectivamente.

4. Es el caso de la torre de la parroquia de Ntra. Sra. de la Encarnación de Constantina. Véase LOPEZ MARTINEZ, Celestino. Desde Jerónimo Hernández... Op. Cit. Págs. 151-152.

5. Archivo Parroquial de San Juan de Marchena (A.P.S.J.M.). Libro $3^{\circ}$. Quentas de Fabrica, Capellanias y Colecturia de los años 1566 asta 1568 inclusive. Fol. 55 vto.

6. El viaje, efectuado por decisión del provisor general del arzobispado, duró tres días y por el mismo cobró Hernán Ruiz 54 reales. Véase nota anterior.

7. La autoría del Hernán Ruiz sobre el campanario de la Iglesia de Santa María de la Mota la di a conocer en MORALES, Alfredo J.: Hernán Ruíz "el Joven". Madrid, 1996. Págs. 74.

8. A.P.S.J.M. Libro $3^{\circ}$. Quentas de Fabrica, Capellanias y Colecturia de los años 1566 asta 1568 inclusive. Fol. 73. 
anterioridad al transcrito, confirman que en 1567 ya se trabajaba en ella ${ }^{9}$. Es más, analizando el concepto correspondiente a varios libramientos se comprueba que el proceso constructivo estaba en aquel momento muy avanzado. Tal circunstancia obliga a adelantar la vinculación de Hernán Ruiz con la obra de la torre de Santa María de la Mota. Sin que por el momento sea posible establecer la fecha precisa, atendiendo a la lentitud de otros procesos constructivos coetáneos, cabe sospechar que el proyecto de campanario para la mencionada iglesia corresponda a unos tres años antes.

No era, pues, la primera vez que Hernán Ruiz se enfrentaba a la edificación de un campanario. Desde que emprendiese la construcción del correspondiente a la Iglesia de San Lorenzo de Córdoba, finalizado en 1555, su experiencia en resolver los problemas compositivos y estructurales de tales edificios se había incrementado, especialmente como consecuencia de su trabajo en la Giralda, que aún no había concluido cuando inició el campanario de Marchena. Como en otras ocasiones, al arquitecto se le presentaba el reto de dotar de un cuerpo de campanas a una torre preexistente. De hecho, la fábrica de la torre de Santa María de la Mota, emplazada a los pies:de la nave del evangelio y adelantada de la linea de muros, ofrece una diversidad de técnicas constructivas que responden a distintos momentos o fases de edificación. Así, en su frente de poniente -único no adosado-, cuenta con un basamento de sillares, de aspecto similar a los empleados en la inmediata portada gótica del astial del templo, actualmente cegada. Sobre aquel, la estrecha caña de la torre apenas supera los cuatro metros por cada lado-, presenta una fábrica con superposición de cajones de tapial, reforzándose sus ángulos con ladrillos. De este mismo material está construido el siguiente sector de la torre, en el que se ha dispuesto una doble hilada en saledizo para, a manera de imposta, marcar el primer cuerpo de la torre. El siguiente, tambien de ladrillos, ofrece al centro un óculo para iluminar la escalera interior, de acceso al cuerpo de campanas. Este arranca de una cornisa volada, construida en sillería perfectamente escuadrada, estando formado por tres cuerpos superpuestos y decrecientes. De ellos, los dos inferiores son de planta cuadrada y el de remate circular. En la construcción de los tres se han combinado la piedra, el ladrillo y el azulejo.

Del análisis de las fábricas se deduce que el cuerpo inferior, con planta cuadrada y aprisionado entre el muro del templo y el del compás del paredaño Convento de la

9. Aunque sin especificar la fecha concreta del pago, son varios los correspondientes a materiales para la construcción de la torre. Así ocurre, por ejemplo, con los efectuados a los tejeros Juan de Estepa y Antón Sánchez, vecinos ambos de Marchena, por diversas partidas de ladrillos para dicha obra. Con los anotados a favor del vecino de Sevilla Gaspar López, por maderas para andamios. Del realizado a los canteros Pedro Francés y Juan Ruiz por labrar "los cartones para la torre". Con varias anotaciones por carretaje de sillares procedentes de Morón y de Osuna. Por varios libramientos a Cristóbal Rodríguez por partidas de cal blanca. A.P.S.J.M. Libro $3^{\circ}$. Quentas de Fabrica, Capellanias y Colecturia de los años 1566 asta 1568 inclusive. Fols. 53, 61, 66, 67 rto. y vto., respectivamente. 
Concepción, es una obra medieval hasta donde alcanza la construcción en tapial ${ }^{10}$. Dicho cuerpo fue reforzado con sillares en su tercio inferior, posiblemente cuando se inició el campanario. Aquel es practicamente macizo -fue parcialmente rozado para ampliar una pequeña habitación abierta al templo-, lo que condicionó la ubicación de la escalera de acceso a los cuerpos superiores. Esta, de acusada pendiente, arranca de un pequeño patio con ingreso desde la nave de la iglesia, desembocando en un estrecho rellano. Del mismo surge una escalera de caracol, alojada en un cuerpo cilíndrico, que se adosa exteriormente a la caña de la torre por su frente oriental. La citada escalera concluye antes de iniciarse el segundo cuerpo de la torre. Tal fórmula, bàstante ingeniosa y enormemente práctica, recuerda la adoptada en las iglesias cuyo astial se resuelve en torre-fachada. Otra escalera, a la que ilumina el óculo abierto en el frente oeste de la torre, se aloja en el segundo cuerpo, enlazando con el caracol encapsulado por el volumen cilíndrico dispuesto en el primer cuerpo de campanas. Toda aquella obra, incluyendo el sector en ladrillos del cuerpo bajo de la torre, es consecuencia directa de la construcción del campanario, correspondiendo por lo tanto al proyecto de Hernán Ruiz II.

El estilo del arquitecto se percibe claramente en la composición y motivos ornamentales del cuerpo de campanas. Este se levanta sobre un zócalo, articulado por pilastrillas, entre las que se han dispuesto recuadros con esquemas geométricos que combinan el ladrillo y la azulejería. Dichos motivos, parecidos a los empleados en el antepecho de la Giralda, van centrados por espejos y puntas de diamante de cerámica vidriada, dispuestos alternativamente, recordando las creaciones y engastes de la joyería coetánea. De este antepecho surge un paralelepípedo, con gruesos pilares en los ángulos apeando arcos de medio punto. Una labor denticulada e hiladas de ladrillos en saledizo marcan la linea de impostas y funcionan a modo de capitel. Idénticos motivos bordean la rosca de los arcos, en cuya clave se disponen ménsulas de piedra. Sobre la superficie de los pilares y mediante azulejos se han organizado unos recuadros, que otorgan a aquellos la apariencia de pilastras cajeadas. La piezas cerámicas organizan un primer tablero de ajedrezado, en los frentes norte, este y oeste, mientras en el flanco sur dicha superficie está ocupada por paneles figurativos con laureas que encierran las armas de Don Luis Cristóbal Ponce de León, Duque de Arcos. Tal ubicación se explica por ser este el frente de la torre visible desde el hoy desaparecido palacio de los señores de la villa ${ }^{11}$. También se emplearon azulejos polícromos para dibujar las enjutas de los arcos, piezas de cerámica cuya presencia simula el cajeado de las superficies. Un volumen cilíndrico, con pequeñas troneras de iluminación, aloja la escalera de caracol que accede al piso superior. Aquel no aparece centrado, sino desplazado hacia el frente occidental, a cuyos machones se adosa.

10. Recientemente se ha señalado que dicho torreón es una obra islámica, dudándose si corresponde a una construcción militar o religiosa. Véase RAVE PRIETO Juan Luis: El Alcázar y la muralla de Marchena. Marchena, 1993. Pág. 94.

11. Véase nota anterior, págs. 109-151. 
Para el cuerpo intermedio del campanario, el arquitecto repitió la misma fórmula compositiva, si bien reduciendo logicamente las proporciones y alterando algunos aspectos omamentales. Este proceso de reducción y concentración dió como resultado una imagen que se aproxima bastante a la que ofrece el Cuerpo del Pozo de la Giralda. El mencionado segundo cuerpo se asienta sobre un zócalo de idénticas proporciones que el primero, presentando recuadros rehundidos y ocupados por paneles de azulejos polícromos en ajedrezado. Los ángulos de dicho zócalo ofrecen pedestales con encintados de azulejería. Retranqueados respecto al basamento se han dispuesto los pilares que apean los arcos de medio punto. Se repiten aquí las hiladas de ladrillo y el denticulado marcando las líneas de imposta y rosca de los arcos, los cuales carecen de ménsulas en la clave. También se recurre a encintados de cerámica vidriada para fingir cajeados en los soportes y enjutas, aunque en este caso se trata de piezas monócromas. En la volada comisa que da paso al remate del campanario se repite el denticulado.

La coronación del cuerpo de campanas la resolvió el arquitecto con un cuerpo cilíndrico. Este ofrece cuatro huecos adintelados, dispuestos sobre los pilares angulares del piso inferior, mientras a eje con los arcos ha situado encintados de cerámica vidriada. Piezas de azulejería sirven, asímismo, para marcar el entablamento que remata el cuerpo y para recubrir el trasdós del casquete esférico de la cubierta. En su composición, el remate del campanario de Marchena, recuerda al que Hernán Ruiz diseñó unos diez años antes para la Iglesia de San Lorenzo de Córdoba ${ }^{12}$. La principal diferencia estriba en la eliminación de las pilastras que ordenan los apoyos y en la sustitución de los elementos figurativos de la torre cordobesa por el contraste de texturas, riqueza cromática y posibilidades expresivas derivada de la sabia combinación de los materiales en la torre marchenera. En este sentido, la torre de Santa María de la Mota es clara consecuencia de la construcción de la Giralda sevillana. No obstante, resulta evidente que la torre de Marchena ha perdido los remates, eolípilas y demas motivos de coronación del segundo cuerpo, elementos que contribuirían a enriquecer plásticamente el conjunto y a dotarlo de un perfil menos rotundo ${ }^{13}$. La presencia de tales elementos, cuya momento de desaparición se ignora, relacionaría más estrechamente este campanario con los otros diseñados por el arquitecto.

Respecto al proceso constructivo de la torre marchenera, ya se anticipó que estaba muy avanzado en 1567 , resultando imposible precisar, por el momento, la exacta fecha de su inicio. Otro tanto ocurre respecto a la conclusión, pues si bien

12. A la organización de ésta pertenece el dibujo del folio $65 \mathrm{vto}$. del Manuscrito de Arquitectura de Hernán Ruiz ПI, como sagazmente identificó Navascués. Véase NAVASCUES PALACIO, Pedro: $E l$ Libro de Arquitectura de Hernán Ruiz el Joven. Madrid, 1974. Págs. 18-19.

13. A ellos puede referirse un pago efectuado al carretero Juan de Lama "por la trayda delharpon ydos seras de azulejos ydosbolas grandes ydoze pequeñas". A. P.S.J.M. Libro $3^{\circ}$. Quentas de Fabrica, Capellanias y Colecturia de los años 1566 asta 1568 inclusive. Fol. 72 vto. Como se verá, dicho harpón fue realizado por Cosme de Sorribas. 
las noticias relativas al primer semestre del año citado son abundantes - podría hablarse casi de nóminas semanales durante algún periodo-, la información se reduce con posterioridad, hasta practicamente desaparecer. Esto podría ser indicio de la conclusión de la torre en ese momento, al menos en sus aspectos fundamentales. En relación con los artistas que intervinieron en la obra, los hay de origen sevillano, habituales colaboradores de Hernán Ruiz, junto a otros de procedencia ignorada y desconocidos hasta el momento. Entre los primeros cabe citar al herrero Cosme de Sorribas, quien trabajaría con el arquitecto en diversas obras para la catedral sevillana ${ }^{14}$. Para la torre de Marchena, Sorribas realizó "unharpon e cruz", piezas metálicas para el remate del campanario por las que percibió 4.746 maravedís ${ }^{15}$. También trabajaron en la catedral, concretamente en la Capilla Real, Juan y Lorenzo Rodríguez, quienes aparecen labrando sillares para el campanario de Santa María de la Mota durante varios meses de $1567^{16}$. El primero en incorporarse a la obra fue Juan, a quien se le nombra unas veces como cantero y otras como entallador. Por su parte Lorenzo aparece en las nóminas semanales a partir del mes de junio, designándosele siempre como cantero. Esta diferenciación en la escala profesional permite identificar a dichos artistas con los hermanos que a finales de 1566 suscribieron contrato de aprendizaje con Hernán Ruiz ${ }^{17}$. Con este y con sus obras en la catedral sevillana colaboró, asímismo, el cantero Juan de Ochoa, cuyo trabajo en la torre marchenera se prolongó, al menos, durante todo el año $1567^{18}$.

Entre los artistas cuya colaboración con el arquitecto se documenta por vez primera y de los que no existían noticias hasta ahora se encuentra el ceramista sevillano Luis de Orellana, quien suministró los azulejos y espejos de cerámica vidriada de la torre ${ }^{19}$. Otro tanto ocurre con el cantero Gonzalo Linar, quien junto a Juan Rodríguez y Juan de Ochoa aparece labrando cantería para el campanario de

14. Sobre esta colaboración puede verse MORALES, Alfredo J.: "La arquitectura de la catedral de Sevilla en los siglos XVI, XVII y XVIII" y MORALES, Alfredo J.:"Artes aplicadas e industriales en la catedral de Sevilla", ambos en La Catedral de Sevilla. Sevilla, 1985. Págs. 199, 200, 201, 563 y 573.

15. A.P.S.J.M. Libro $3^{\circ}$. Quentas de Fabrica, Capellanias y Colecturia de los años 1566 asta 1568 inclusive. Fol. 70 vto. Para "poner el harpon ypiedra bola enla bobeda dela torre" se compraron al calderero Juan López, vecino de Marchena, siete libras de plomo. A.P.S.J.M. Idem. Fol. 72.

16. Véase MORALES, Alfredo J.: La Capilla Real ... Op. Cit. Págs. 94 y 96.

17. Dicho contrato fue publicado por LOPEZ MARTINEZ, Celestino: Desde Jerónimo Hernández ... Op. Cit. Pág. 136. Para más información sobre el mismo y sobre la distinta cualificación de los hermanos Rodríguez, véase MORALES, Alfredo J.: Hernán Ruiz ... Op. Cit. Págs. 138-139.

18. En 1551 figura un entallador del mismo nombre y apellido en las nóminas de la catedral sevillana. Véase GESTOSO Y PEREZ, José Ensayo de un diccionario de los artífices que florecieron en Sevilla desde el siglo XIII al XVIII inclusive. Tomo I. Sevilla, 1899. Pág. 195. Posiblemente sea el mismo artista que trabajó en la construcción del Ayuntamiento hispalense. Al respecto, MORALES, Alfredo J.: "El Ayuntamiento de Sevilla: maestros canteros, entalladores e imagineros", en Laboratorio de Arte. $\mathrm{N}^{\circ} 4$. 1991. Pág. 73. A pesar de la coincidencia onomástica no se trata del arquitecto cordobés que sucedería a Hernán Ruiz II en algunas de sus obras en tierras de Córdoba.

19. A.P.S.J.M. Libro $3^{\circ}$. Quentas de Fabrica, Capellanias y Colecturia de los años 1566 asta 1568 inclusive. Fol. 73 vto. 
Marchena entre abril y mayo de $1567^{20}$. Asímismo, con los maestros albañiles Diego López Pavón y Juan de Morales, quienes en su trabajo fueron ayudados por diversos peones, si bien las labores más cualificados, como cortar y preparar los ladrillos y azulejos, les correspondieron a ellos ${ }^{21}$.

La torre de Santa María de la Mota de Marchena, hasta aquí analizada, es sin duda un hito destacado en el camino de especialización sobre campanarios desarrollado por Hernán Ruiz el Joven. De su estudio se desprende que el arquitecto supo siempre acomodarse a las exigencias de las obras preexistentes y que fue capaz de resolver los innegables problemas estructurales implícitos a tales edificaciones. Por otra parte se comprueba su genialidad para recurrir a un mismo lenguaje formal, sin caer en la monotonía o la reiteración. Es más, en las variaciones sobre fórmulas compositivas y soluciones ornamentales ya experimentadas radica gran parte de su genio y maestría.

20. Aparece por última vez en una nómina del 27 de mayo, ocupando su lugar en las siguientes partidas, desde el 18 de junio, Lorenzo Rodríguez. A. P. S. J. M. Libro $3^{\circ}$. Quentas de Fábrica, Capellanías y Colecturia de los años 1566 asta 1568 inclusive. Fols. 90 y 93 vto., respectivamente.

21. Idem. Fol. 119 vto y ss. 

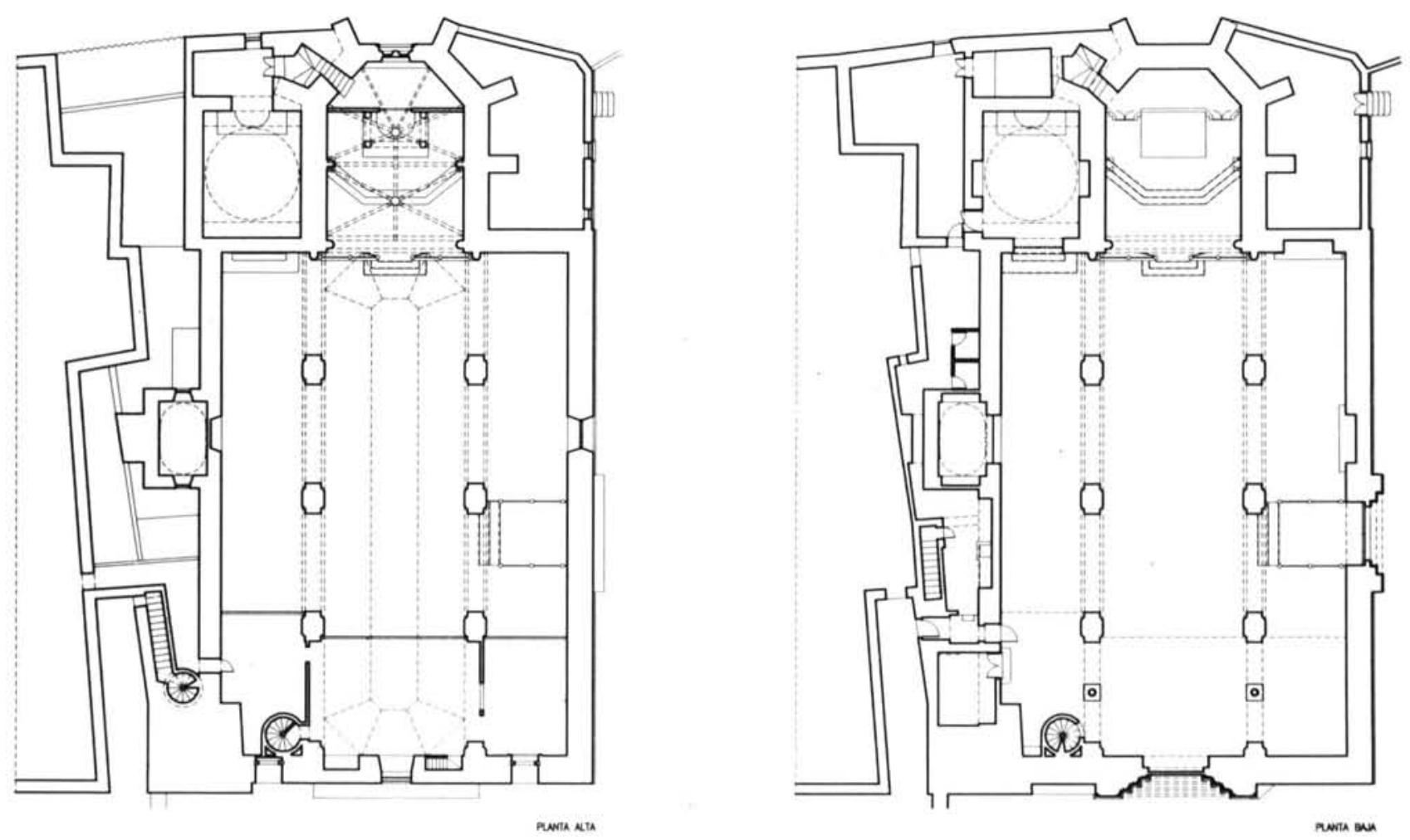

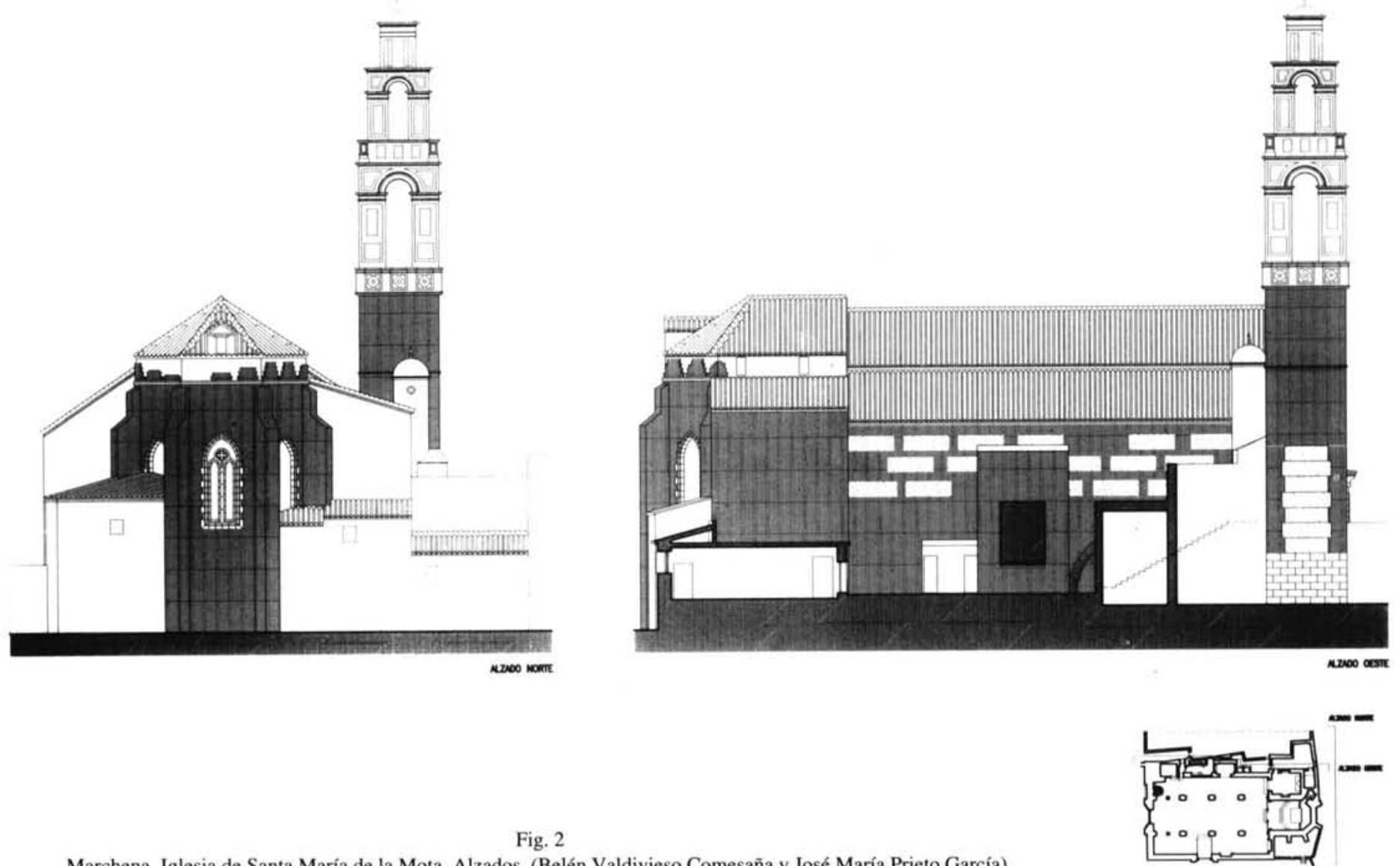


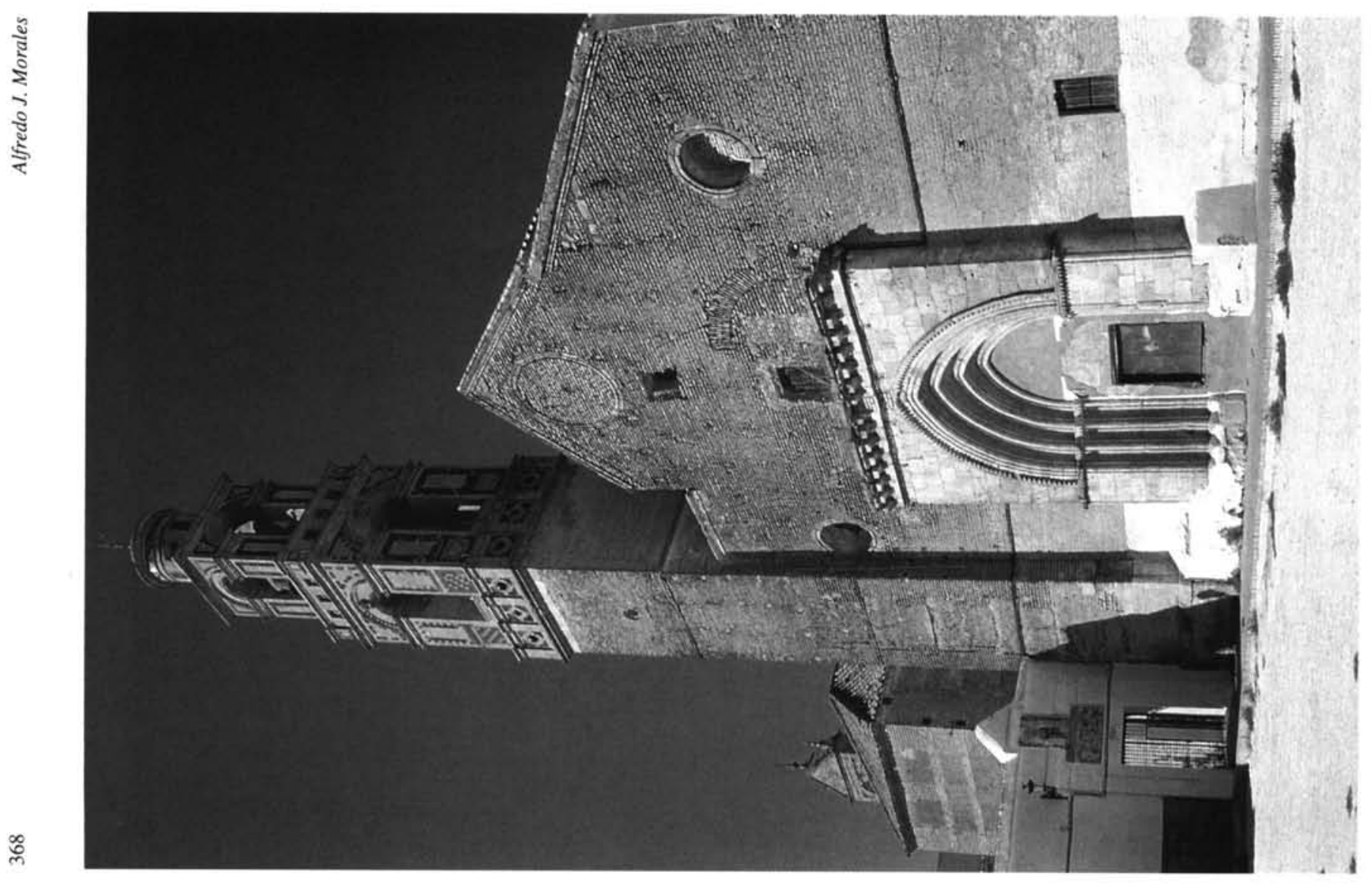




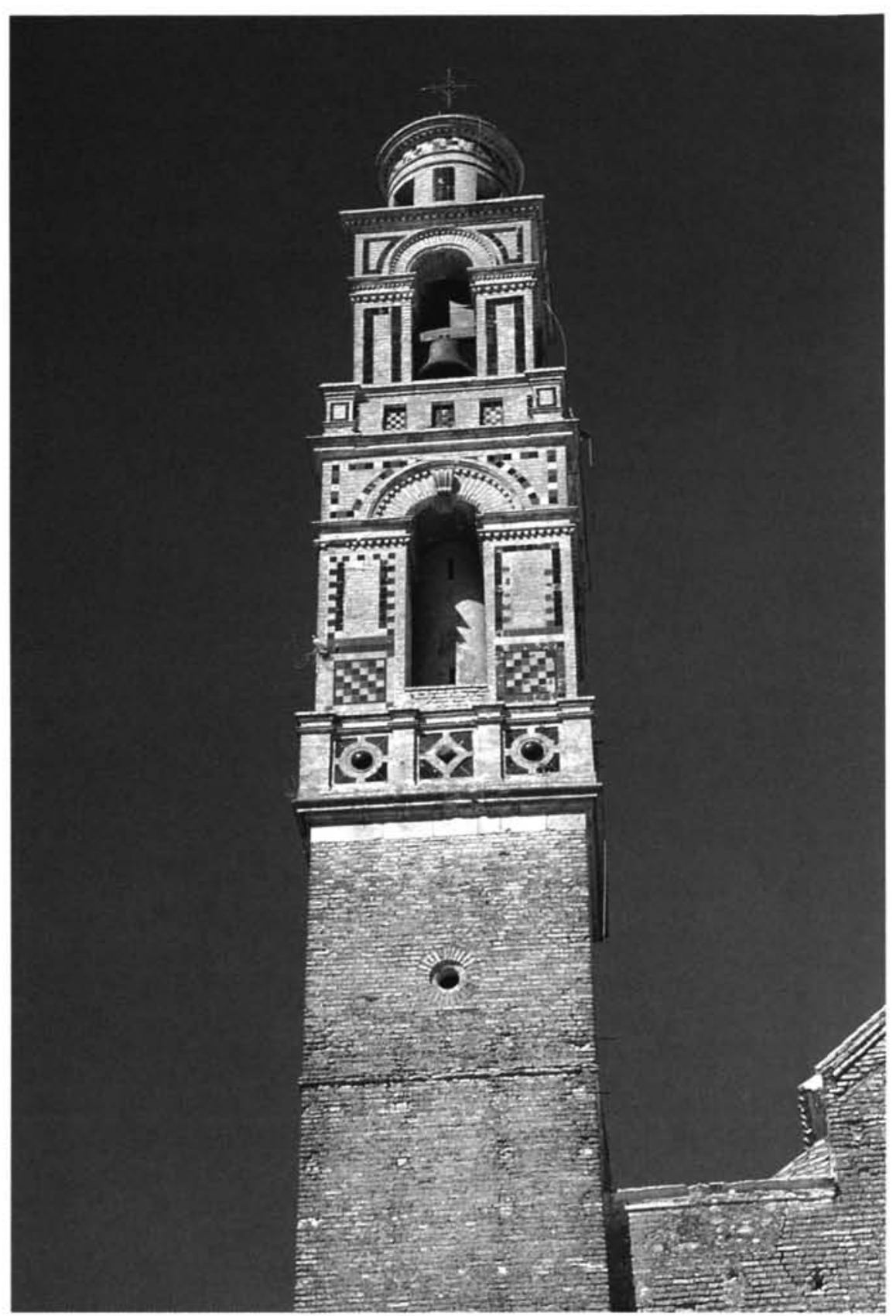

Fig. 4

Marchena. Iglesia de Santa María de la Mota. Torre. Hernán Ruiz el Joven. 